\title{
Dexmedetomidina en dolor visceral
}

\section{Dexmedetomidine in visceral pain}

\author{
Catalina Kychenthal'1 , Cristóbal López¹, Francisca Elgueta²
}

\begin{abstract}
Visceral pain is a common symptom amongst the population, both in acute and chronic presentations. It is the result of multiple mechanisms and complex interactions, which to this day are not fully understood. Due to this, there are no specific treatment options available, and it is usually managed with opioids, NSAIDs, antispasmodics and antidepressants. There have recently been studies which contemplate the use of dexmedetomidine as management for visceral pain. This drug is an alpha-2 adrenergic agonist with sedative, analgesic, anxiolytic and sympatholytic properties, besides a good safety profile with minimal respiratory depression and little hemodynamic repercussion. The available studies suggest that this drug reduces visceral pain significatively through multiple mechanisms, reduces opioid consumption and has high patient satisfaction without an important raising of side effects. In spite of looking as a promising alternative, there are not enough studies or substantial evidence to categorically recommend the use of dexmedetomidine for the management of visceral pain. With further research, in the future this could turn into an important alternative for analgesic schemes in these patients.
\end{abstract}

\section{RESUMEN}

El dolor visceral es un síntoma prevalente en la población, tanto en su forma aguda como crónica. Este es el resultado de diversos mecanismos e interacciones complejas, las cuales hasta el día de hoy no son completamente comprendidas. Debido a esto, no existen tratamientos específicos para este y se maneja clásicamente con opioides, AINEs, antiespasmódicos y antidepresivos. Recientemente, se ha estudiado el uso de la dexmedetomidina para el manejo del dolor visceral. Este fármaco es un alfa-2 agonista con propiedades sedantes, analgésicas, ansiolíticas y simpaticolíticas, que además posee un buen perfil de

\section{Key words:}

Dexmedetomidine, visceral pain, nociceptive pain, analgesia

\section{Palabras clave:}

Dexmedetomidina, dolor visceral, dolor nociceptivo, analgesia

\section{Facultad de Medicina, Pontificia Universidad Católica de Chile. Santiago, Chile.}

Departamento de Anestesiología, Facultad de Medicina, Pontificia Universidad Católica de Chile. Santiago, Chile.

Fecha de recepción: 30 de julio de 2020

Fecha de aceptación: 28 de agosto de 2020

\section{ORCID}

0000-0001-9507-2688

\section{Correspondencia:}

Francisca Elgueta

elguetafrancisca@yahoo.com 
seguridad con baja repercusión hemodinámica y en la mecánica respiratoria. Los estudios que existen a la fecha acerca de la relación entre este fármaco y el dolor visceral sugieren una mejora significativa de la sintomatología por diversos mecanismos, con reducción de la necesidad de rescates con opioides y buena satisfacción usuaria sin un alza importante de efectos adversos. A pesar de parecer prometedor, faltan estudios y evidencia para recomendar de manera categórica el uso de dexmedetomidina con este propósito, pudiendo ser en el futuro una buena alternativa para considerar en el manejo de estos pacientes.

\section{Introducción}

E I dolor visceral (DV) es un problema altamente prevalente y con grandes efectos en la calidad de vida de los pacientes, sobre todo cuando se trata de sintomatología crónica. Los mecanismos de acción de este no están completamente dilucidados, debido a lo cual no existen esquemas de manejo analgésico específico para abordarlo[1].

Actualmente, existen estudios que sugieren un efecto beneficioso del uso de dexmedetomidina, un agonista alfa-2 adrenérgico, en pacientes que sufren de dolor visceral. Esto es todavía un campo en estudio, y no existen guías que lo recomienden ni literatura que avale esta conducta terapéutica. En este contexto, este trabajo pretende investigar esta relación, y de esa manera abrir paso para su posible empleo en el esquema analgésico de estos pacientes.

\section{Dexmedetomidina}

La dexmedetomidina es un fármaco agonista altamente selectivo del receptor adrenérgico alfa-2. Este posee diversos usos, entre los cuales destaca la sedación, analgesia, ansiolisis y efectos simpaticolíticos. Su mecanismo de acción se basa en la gran afinidad para activar y modular los receptores mencionados, los cuales se ubican en sitios pre, post y extra sinápticos a través de todo el cuerpo. En el sistema nervioso central (SNC) actúa principalmente disminuyendo la concentración de norepinefrina local, hiperpolarizando membranas neuronales y participando de la modulación de estímulos nociceptivos[2]. La dexmedetomidina es una molécula lipofílica con alto grado de unión a proteínas plasmáticas y vida media de aproximadamente 2 h, dependiente de metabolización hepática y excreción renal. Estas características se traducen en un rápido inicio de acción, corta duración de sus efectos y un buen perfil de seguridad[3]. Este fármaco puede ser administrado de forma intravenosa, oral, sublingual, intranasal e intramuscular.

Los principales escenarios en los cuales se utiliza la dexmedetomidina incluyen las unidades de cuidados intensivos $(\mathrm{UCl})$, ambientes perioperatorios y en pacientes pediátricos. Dentro de sus utilidades en las $\mathrm{UCl}$, destaca por su efectividad para sedación a corto y largo plazo en pacientes conectados a ventilación mecánica invasiva, con mayor facilidad para despertar y menor incidencia de agitación y delirium[4]. En contextos quirúrgicos, la dexmedetomidina es una buena alternativa en pacientes con mala tolerancia a potenciales deterioros cardiorrespiratorios, dado su estabilidad hemodinámica y mínima depresión respiratoria[2]. Además, su uso perioperatorio como adyuvante a la anestesia general y analgésicos es de suma utilidad, debido a que reduce los requerimientos de opioides, la presencia de náuseas y vómitos postoperatorios y de prurito, sin aumentar los efectos secundarios[5]. Debido a su amplio espectro de presentaciones, también es utilizado como ansiolítico en la pre-medicación de pacientes pediátricos, agitados o sin disponibilidad de vías intravenosas. En ambientes pediátricos es utilizado debido a su buen perfil de seguridad y a su capacidad de reducir la cantidad de otros fármacos administrados, por ende reduciendo los efectos adversos. Esto tiene relevancia principalmente en el manejo intensivo de dichos pacientes, o en aquellos que debido a condiciones crónicas requieren múltiples sedaciones, particularmente, los niños con déficits neurológicos[6].

Cabe destacar que, de todas formas, la dexmedetomidina puede asociarse a efectos adversos graves. A pesar de su mínimo efecto en la mecánica ventilatoria, esta podría llevar a la obstrucción de la vía aérea, sobre todo al combinarse con otros fármacos con este potencial. Con respecto a efectos hemodinámicos, estos son dependientes de la dosis. A dosis bajas, el aumento de actividad parasimpática puede llevar a hipotensión, y a dosis altas podría causar hipertensión transitoria seguida de bradicardia e hipotensión refleja[7]. Debido a esto, su uso siempre debe 
monitorizarse y considerarse individualmente según las condiciones de cada paciente.

\section{Dolor visceral}

El dolor visceral es un dolor originado en órganos internos, que suele presentarse como dolor difuso y acompañarse de sintomatología neurovegetativa. Es una patología muy prevalente y causa de importante deterioro de la calidad de vida, además de contar con grandes consecuencias económicas secundarias a ello[8]. Tiene múltiples causas agudas tales como la apendicitis, colecistitis y úlcera péptica; y también crónicas como las patologías funcionales - incluyendo el síndrome de intestino irritable (SII) - y la dismenorrea.

\section{Mecanismos de dolor visceral}

Los mecanismos tras el dolor visceral son complejos y constan de una amplia gama de componentes, incluyendo distintos tipos de aferencias y el procesamiento central de estas, además de elementos emocionales, mediadores inflamatorios, microbiota intestinal y otros.

Existen similitudes entre algunos de los mecanismos por los cuales se percibe el DV con aquellos del dolor somático, pero difieren en diversos aspectos en cuanto al procesamiento de este. Un ejemplo es la falta de distinción clara entre aferencias nociceptivas y no-nociceptivas, las cuales son percibidas de forma similar al provenir del compartimento visceral pero están firmemente diferenciadas en el dolor somático. Existen, además, características complejas que explican el carácter difuso del dolor visceral, como lo son la baja densidad relativa de inervación, la convergencia de aferencias y la ramificación de los axones. Estas explican también el origen del dolor referido como presentación alternativa de DV[1].

La fisiopatología del DV radica en la interpretación de que los nociceptores han entrado en contacto con potenciales noxas para el sistema, las cuales pueden originarse en la distensión de órganos huecos, hipoxia tisular, mediadores de inflamación local, etc. Existen ocasiones, sin embargo, en que estímulos que no son nocivos son interpretados de esta forma, lo cual es la base que explica los trastornos funcionales gastrointestinales crónicos como lo es el SII. La explicación de esto puede estar en distintos niveles e incluye la sensitización de terminales periféricas, sensitización central y cambios en la modulación a nivel central del dolor secundarios a estados de estrés emocional[9].

Existe un modelo llamado el eje cerebro-intesti- nal, que ilustra la red de conexiones nerviosas que conectan los centros emocionales, cognitivos y autonómicos del SNC con el sistema nervioso entérico (SNE), centros neuroendocrinos y el sistema inmune. La teoría apunta a que cualquier alteración de estas interacciones puede ser causa de diversas desregulaciones y por ende cuadros agudos o crónicos de dolor visceral[1].

\section{Manejo de dolor visceral}

De manera global y como fue mencionado, el DV puede ser explicado por la sensitización de aferencias viscerales, la modulación central que lleva a la amplificación del dolor o la mezcla de ambas. Al ser difícil de identificar, no existen tratamientos específicos para este dolor hoy en día. Lo más utilizado son los opioides, cuyo uso está limitado por sus efectos adversos (incluyendo constipación), desarrollo de tolerancia, hiperalgesia inducida por opioides o dependencia. En este contexto, pueden utilizarse antiinflamatorios no esteroidales (AINEs), paracetamol, antiespasmódicos y antidepresivos, particularmente inhibidores de la recaptura de serotonina[10]. Comúnmente, el DV se ve estrechamente relacionado a estados afectivos tales como la depresión o la ansiedad, y por ende el manejo de estas debe considerarse como un pilar fundamental en el abordaje de estos cuadros[11].

\section{Uso de dexmedetomidina en dolor visceral}

Recientemente, existen reportes en la literatura que asocian el uso de dexmedetomidina a una reducción significativa de dolor visceral en distintos escenarios.

Un estudio randomizado analizó a pacientes sometidos a cirugía abdominal laparoscópica, comparando el uso de oxicodona sola o asociada a dexmedetomidina. Los autores observaron que el apoyo con dexmedetomidina se relacionaba con menor dolor visceral objetivado con escala visual análoga (EVA), menor necesidad de rescates de analgesia y mayores niveles de satisfacción de los pacientes en cuanto a manejo del dolor. Esto no significó mayores efectos adversos, exceptuando el grupo con altas dosis de dexmedetomidina en que se evidenció mayor ocurrencia de hipotensión, sin que esta se asociara a episodios de bradicardia[12].

Otro contexto en el cual se ha utilizado este fármaco para el manejo de dolor visceral agudo es en múltiples estudios prospectivos de ratas expuestas a sustancias inflamatorias viscerales. En ellas, se ha evi- 
denciado un efecto analgésico significativo mediante la reducción de señales nociceptivas y resultante supresión de hipersensibilidad visceral[13]-[15].

Se ha reportado también el uso de este fármaco en DV crónico oncológico de difícil manejo. En el caso descrito por Seymore et al., el uso de dexmedetomidina significó la reducción más significativa de requerimiento de opioides al usarse como adyuvante de estos, comparándose con otras drogas incluyendo lidocaína, ketamina, midazolam y metadona[16].

\section{Mecanismos y fisiopatología}

Se han formulado diferentes teorías en cuanto a los mecanismos que pueden explicar esta relación. Uno de los posibles ejes de acción por el cual la dexmedetomidina regularía el dolor visceral es mediante el bloqueo de la extracellular signal-regulated kinase (ERK) en la vía MEK (mitogen-activated extracellular signal-regulated kinase) - ERK - CREB (CAMP response element binding protein kinase). Se ha estudiado que esta vía participa en la transmisión, modulación y mantención del dolor visceral, por lo que su bloqueo resultaría en propiedades analgésicas[17],[18].

Li Sun et al., observó que al inducir DV a ratas, la administración de dexmedetomidina se asociaba a un aumento de miR-21, el cual suprime la activación de ERK y su vía de señalización, regulando así el dolor visceral generado[14].

Se ha demostrado también que junto a la modulación de la vía de señalización asociada a ERK, la dexmedetomidina también tiene un rol mediante nocicepción asociada a Toll-like receptors (TLR) y el canal receptor de potencial transitorio (TRP). En ratas a las que se les administró dexmedetomidina, disminuyó la activación de ERK, TL4 y en el canal TRPV1, lo que se vió reflejado en una disminución del dolor[13].

Similar al estudio realizado por Li Sun et. al, Meng Liang et al., realizaron un trabajo en ratas donde observaron otro posible eje de acción de la dexmedetomidina. En esta investigación, demostraron que al administrar ácido 2,4,6-Trinitrobencenosulfónico e inducir dolor visceral hubo una disminución en la expresión de miR-34a, el cual se ha asociado de distintas formas a procesos inflamatorios y deletéreos en el organismo. Se estipula la existencia de una modulación del dolor a través de miR-34a y su relación inhibiendo la expresión del gen HDAC2. Al administrar dexmedetomidina, se restauró la expresión de miR-34a en las ratas, lo cual fue reflejado como disminución del dolor visceral[19].

Lan et al., indujo dolor visceral en ratas mediante ácido acético y evaluó la respuesta a la administración de dexmedetomidina, encontrando signos de control de dolor asociados al uso del fármaco. Ellos proponen como mecanismo de modulación del dolor mediante la vía Keap1/Nrf2, eje que ha sido estudiado como modulador de la respuesta a estrés oxidativo y procesos inflamatorios asociados. Su hipótesis se sustenta en el aumento de señalización de esta vía tras la administración de ácido acético, la cual fue regulada posteriormente mediante el uso dexmedetomidina[20].

Dado la amplia distribución de los receptores alfa2 adrenérgicos, es de interés analizar a qué nivel se produce la reacción asociada a la analgesia. Debido a esto, en ratas con DV inducido y posterior manejo con dexmedetomidina, se administró un antagonista de dicho receptor a nivel periférico (MK-467). Esta intervención no se tradujo en una reducción del efecto analgésico, por lo que se concluyó que el efecto principal no se encuentra asociado a los receptores ubicados a nivel periférico[21].

En el trabajo realizado por Zhang et al., con DV inducido por formaldehído en ratas, el efecto de la dexmedetomidina se asoció a inhibición en la expresión de nNOS, PKCg y PAR2. Este efecto era reversible mediante el la administración de yohimbina e idazoxano, concluyendo que el efecto analgésico de la dexmedetomidina es mediado tanto por el receptor alfa-2 adrenérgico como por el receptor de imidazolina[22].

\section{Discusión}

En suma, la dexmedetomidina pareciera tener un efecto benéfico en el manejo de dolor visceral tanto agudo como crónico en pacientes que sufren de esta condición, y puede ser considerada como alternativa analgésica. Fisiopatológicamente, existen distintas explicaciones para este fenómeno que parecen consistentes tanto con los conocimientos previos de este fármaco y del DV como con el resto de los estudios que abordan el mismo tema.

Esta relación podría tener grandes implicancias, debido a la gran cantidad de pacientes que sufren de DV ya sea por cirugías abdominales, enfermedades de curso agudo o condiciones crónicas tanto oncológicas como benignas. Destaca además el buen perfil de seguridad que posee esta droga, ya que la vuelve una buena opción a la hora de utilizar adyuvancia en pacientes recientemente sometidos a anestesia general, cursando cuadros o descompensaciones agudas o aquellos que requieren de un fármaco seguro para utilizar a permanencia. En el caso crónico, el uso potencial de dexmedetomidina posee un beneficio adicional al tratar con un dolor clásicamente manejado 
con opioides, en el contexto de la creciente dependencia a estos que se observa en la población.

A pesar de que los resultados parecen ser prometedores, existe todavía muy poca evidencia para respaldar su uso de forma categórica. La evidencia existente posee además diversas limitaciones, como por ejemplo la heterogeneidad de los químicos utilizados en los estudios de DV en ratas, lo cual dificulta la comparación de sus resultados. Es por esto que se necesitan más estudios y evidencia de buena calidad para recomendar el uso de la dexmedetomidina en estos contextos clínicos. Cabe recalcar la relevancia de tratar de forma individualizada a cada paciente, tomando en cuenta los beneficios y los riesgos de cada uno a la hora de seleccionar el esquema analgésico a utilizar.

\section{Referencias}

1. Sikandar S, Dickenson A. Visceral pain. Current Opinion in Supportive and Palliative Care. 2012;6(1):17-26. https://doi.org/10.1097/ SPC.0b013e32834f6ec9

2. Nguyen $V$, Tiemann D, Park E, Salehi A. Alpha-2 Agonists. Anesthesiology Clinics. 2017;35(2):233-245. https:// doi.org/10.1016/j.anclin.2017.01.009

3. Davy A, Fessler J, Fischler M, Le Guen M. Dexmedetomidine and general anesthesia: a narrative literature review of its major indications for use in adults undergoing non-cardiac surgery. Minerva Anestesiologica. 2017; 83(12):1294-308

4. Keating G. Dexmedetomidine: A Review of Its Use for Sedation in the Intensive Care Setting. Drugs. 2015;75(10):1119-1130. https://doi.org/10.1007/s40265015-0419-5

5. Peng K, Liu H, Wu S, Cheng $\mathrm{H}$, Ji F. Effects of Combining Dexmedetomidine and Opioids for Postoperative Intravenous Patient-controlled Analgesia. The Clinical Journal of Pain. 2015;31(12):1097-1104. https://doi.org/10.1097/ AJP.0000000000000219

6. Buck M, Willson D. Use of Dexmedetomidine in the Pediatric Intensive Care Unit. Pharmacotherapy. 2008;28(1):51-57. https:// doi.org/10.1592/phco.28.1.51

7. Weerink $M$, Struys $M$, Hannivoort L, Barends C, Absalom A, Colin P. Clinical Pharmacokinetics and Pharmacodynamics of Dexmedetomidine. Clinical Pharmacokinetics. 2017;56(8):893913. https://doi.org/10.1007/ s40262-017-0507-7

8. Grundy L, Erickson A, Brierley S. Visceral Pain. Annual Review of Physiology. 2019;81(1):261-284. https://doi.org/10.1146/annurevphysiol-020518-114525

9. Gebhart G, Bielefeldt K. Physiology of Visceral Pain. Comprehensive Physiology. 2016;6:1609-1633. https://doi. org/10.1002/cphy.c150049

10. Johnson A, Greenwood-Van Meerveld B. The Pharmacology of Visceral Pain. Pharmacological Mechanisms and the Modulation of Pain. 2016;75:273301. https://doi.org/10.1016/ bs.apha.2015.11.002

11. Meerveld B, Johnson A. Mechanisms of Stress-induced Visceral Pain. Journal of Neurogastroenterology and Motility. 2018;24(1):7-18. https://doi. org/10.5056/jnm 17137

12. Jiang Z, Zhou G, Song Q, Bao C, Wang $\mathrm{H}$, Chen Z. Effect of Intravenous Oxycodone in Combination With Different Doses of Dexmedetomidine on Sleep Quality and Visceral Pain in Patients After Abdominal Surgery. The Clinical Journal of Pain. 2018;34:1126-1132. https://doi.org/10.1097/

AJP.0000000000000645

13. Liu $Y$, Liu W, Wang $X$, Wan $Z$, Liu $Y$, Leng $Y$. Dexmedetomidine Relieves Acute Inflammatory Visceral Pain in Rats through the ERK Pathway, Toll-Like Receptor Signaling, and TRPV1 Channel. Journal of Molecular Neuroscience. 2018;66(2):279-290. https:// doi.org/10.1007/s12031-0181172-5

14. Sun L, Zhou J, Sun C. MicroRNA211-5p Enhances Analgesic Effect of Dexmedetomidine on Inflammatory Visceral Pain in Rats by Suppressing ERK Signaling. Journal of Molecular Neuroscience. 2019;68(1):19-28. https://doi.org/10.1007/s12031019-01278-z

15. Zhang $H$, Yan $X$, Wang $D$, Leng $Y$, Wan Z, Liu $Y$ et al. Corrigendum to "Dexmedetomidine relieves formaldehyde-induced pain in rats through both $\alpha 2$ adrenoceptor and imidazoline receptor" [Biomed. Pharmacother. 90 (2017) 914-920]. Biomedicine \& Pharmacotherapy. 2017;96:1569. https://doi.org/10.1016/j. biopha.2017.08.080

16. Seymore R, Manis M, Coyne P. Dexmedetomidine Use in a Case of Severe Cancer Pain. Journal of Pain \& Palliative Care Pharmacotherapy. 2019;33(1-2):34-41. https://doi.org/10.1080/1536028 8.2019.1629520

17. Yu CG, Yezierski RP Activation 
of the ERK1/2 signaling cascade by excitotoxic spinal cord injury. Brain Res Mol Brain Res. 2005;138(2): 244-255. https:// doi.org/10.1016/j.molbrainres.2005.04.013

18. Lai HH, Qiu CS, Crock LW y cols. Activation of spinal extracellular signal-regulated kinases (ERK) $1 / 2$ is associated with the development of visceral hyperalgesia of the bladder. Pain. 2011;152(9):2117-2124. https://doi.org/10.1016/j. pain.2011.05.017

19. Liang M, Shao A, Tang $X$, Feng M, Wang J, Qiu Y. MiR-34a affects dexmedetomidineinhibited chronic inflammatory visceral pain by targeting to HDAC2. BMC Anesthesiol. 2019;19(1):131. https://doi. org/10.1186/s12871-019-0801-z

20. Lan J, Zheng J, Feng J, Peng W. Nrf2 mediates the antinociceptive activity of dexmedetomidine in an acute inflammatory visceral pain rat model by activating the NF- $\kappa \mathrm{B}$ sensor. Cell Biochem Funct. 2020;38(1):97-105. https://doi.org/10.1002/cbf.3456

21. Ulger F, Bozkurt A, Bilge SS, et al. The antinociceptive effects of intravenous dexmedetomi- dine in colorectal distensioninduced visceral pain in rats: the role of opioid receptors. Anesth Analg. 2009;109(2):616622. https://doi.org/10.1213/ ane.0b013e3181a9fae2

22. Zhang $H$, Yan $X$, Wang DG, et al. Dexmedetomidine relieves formaldehyde-induced pain in rats through both $\alpha 2$ adrenoceptor and imidazoline receptor [published correction appears in Biomed Pharmacother. 2017 Nov 15;:]. Biomed Pharmacother. 2017;90:914920. https://doi.org/10.1016/j. biopha.2017.04.047 